\title{
Evolving Role of MRI in Assessment of Response of Hepatocellular Carcinoma Patients Post Trans-Arterial Chemoembolization: What Does the Physician Want to Know?
}

\author{
MOHAMED F.H. ABDALLAH, M.D.; MOHAMED HOSNI K. ABDELMAKSOUD, M.D.; \\ SHIMAA H.I. DESOUKY, M.D.; MONA HASSAN, M.D. and MEDHAT MADBOULY, M.D.
}

The Department of Radiology, Theodor Bilharz, Research Institute, Giza, Egypt

\begin{abstract}
Background: In clinical practice, Transarterial Chemoembolization (TACE) has been widely used for the treatment of Hepatocellular Carcinoma (HCC) beyond as well as within guideline recommendations. Computed Tomography (CT) and Magnetic Resonance Imaging (MRI) play critical roles for assessing treatment response of Hepatocellular Carcinoma (HCC) after locoregional therapy. Interpretation is challenging because post-treatment imaging findings depend on the type of treatment, magnitude of treatment response, time interval after treatment, and other factors.
\end{abstract}

Aim of Study: To illustrate the prime role of MRI in accurate assessment and early monitoring of hepatocellular carcinoma response to treatment after transarterial chemoembolization.

Patients and Methods: This study included 80 patients, 52 males and 28 females, patients ages ranged from 45 to 81 years with the mean age of 60 years underwent transarterial chemoembolization over a period of 23 months (Jan. 2019Dec. 2020). All patients had liver cirrhosis related to chronic viral hepatitis. MRI was conducted at MRI unit in a private radiology centre.

Results: Showed superior diagnostic performance of dynamic MRI compared to diffusion studies as dynamic MRI had a sensitivity of $90.9 \%$, a specificity of $95.7 \%$, PPV of $93.7 \%$, NPV of $93.7 \%$ and overall agreement of $94 \%$ compared to $100 \%, 65.2 \%, 68 \%, 100 \%$ and $80 \%$ respectively of diffusion weighted imaging. The difference between the malignant residual and well-ablated groups ADC variables was statistically significant $p$-value 0.006 .

Conclusion: MRI is a robust tool in detection of tumour viability after TACE of hepatocellular carcinoma and should be performed at regular time intervals. Imaging protocol should include dynamic study combined with diffusion imaging. DW MR imaging is a rapid promising technique for the non-invasive evaluation of tumor response after TACE particularly when contrast medium administration is contraindi-

Correspondence to: Dr. Mohamed F.H. Abdallah, The Department of Radiology, Theodor Bilharz Research Institute, Giza, Egypt cated. Dynamic study is the corner stone in detection of recurrent lesions.

Key Words: MRI - Hepatocellular carcinoma - Chemoembolization.

\section{Introduction}

HEPATOCELLULAR Carcinoma (HCC) is the fifth most common malignancy in the world and the third most common cause of cancer death, with 600,000 to 1 million new cases diagnosed each year [1]

Transarterial Chemoembolization (TACE) of Hepatocellular Carcinoma (HCC) is used in some cases as a bridge to liver transplantation. It is also used for patients with unresectable HCC, and has been shown to improve survival [2]

Early assessment of the effectiveness of TACE is critical in planning future therapy whether earlier treatment of residual viable portions of the tumor, delaying re-treatment in cases of good response to avoid unnecessary toxicity, or switching to a different local-regional therapy approach [3].

Although post-treatment follow-up is usually done with both computed tomography and Magnetic Resonance Imaging (MRI), MRI is found to be superior in detecting residual or recurrent tumors after treatment [4].

The advantages of MR imaging in the investigation of liver disease includes the lack of ionizing radiation of MR imaging and the safety of gadolinium chelates. Unlike iodinated contrast agents, gadolinium chelates are much less likely to result in major allergic reactions. Moreover, recent developments in MR imaging, including fast scanning techniques enable improved detection and charac- 
terization of many liver lesions so that a definitive diagnosis can be made noninvasively. The present study aims to illustrate the growing role of MRI in accurate assessment and early monitoring of hepatocellular carcinoma response to treatment after transarterial chemoembolization [5].

\section{Patients and Methods}

This retrospective study was performed on 80 cases of HCC who underwent hepatocellular carcinoma over a period of 23 months (Jan. 2019December 2020). The study was conducted in the Radiology Department at a private Radiology Center. The patients' ages ranged between 45 to 81 years (mean age 60); 52 patients were males and 28 patients were females. Most of the patients had liver cirrhosis related to chronic viral hepatitis.

\section{Inclusion criteria:}

Patients included in the study were those patients who were candidates for radiofrequency ablation \& already underwent the procedure: Both sexes are included. No age group predilection.

\section{Exclusion criteria:}

Patients with MRI incompatible devices such as cardiac pacemaker, metallic valves, hearing aids or aneurismal clips. Patients with high serum creatinine (above $1.4 \mathrm{mg} \%$ ), for fear of contrast induced nephrogenic systemic fibrosis.

All cases had been subjected to: Full clinical assessment, revision of the patient's laboratory investigations including renal function tests (blood urea and serum creatinine), revision of the radiological investigations previously done for the patients, patients were scheduled to undergo MRI within 1 to 3 months after TACE. In case of absent evidence of residual/recurrent lesions, follow-up arranged to be after 2 to 3 months from the first MRI, positive cases were scheduled to repeat the locoregional therapy.

\section{MRI protocol:}

45 cases were performed using Philips 3 Tesla MRI scanner (Ingenia) and the other 35 cases were performed using Philips open 1 Tesla MRI scanner (Panorama HFO).

\section{A- Pre-contrast imaging:}

I- Coronal ultrafast spin-echo sequence (single breath-hold), which serves as a localizer and provides an overview of the anatomy.

II- Conventional MR sequences and parameters protocol were done as shown in (Table 1).

Table (1): Conventional MRI sequences and parameters.

\begin{tabular}{|c|c|c|c|c|c|c|c|c|c|c|}
\hline \multirow{2}{*}{ Sequence } & \multicolumn{2}{|c|}{ TR (msec) } & \multicolumn{2}{|c|}{$\mathrm{TE}(\mathrm{msec})$} & \multicolumn{2}{|c|}{ FOV (mm) } & \multicolumn{2}{|c|}{ Flip angle } & \multicolumn{2}{|c|}{ Slice thickness } \\
\hline & $1 \mathrm{~T}$ & $1.5 \mathrm{~T}$ & $1 \mathrm{~T}$ & $1.5 \mathrm{~T}$ & $1 \mathrm{~T}$ & $1.5 \mathrm{~T}$ & $1 \mathrm{~T}$ & $1.5 \mathrm{~T}$ & $1 \mathrm{~T}$ & $1.5 \mathrm{~T}$ \\
\hline - Axial T1 turbo field echo (TFE) & 14 & 10 & 6.9 & 4.6 & \multicolumn{2}{|c|}{$300-350$} & 15 & 15 & & \\
\hline - Axial T2 turbo spin echo (TSE) & 1000 & 1000 & 80 & 80 & \multicolumn{2}{|c|}{$300-350$} & 90 & 90 & & \\
\hline $\begin{array}{l}\text { - Axial T2 spectral attenuated inversion } \\
\text { recovery (SPAIR) }\end{array}$ & 1000 & 1000 & 80 & 80 & \multicolumn{2}{|c|}{$300-350$} & 90 & 90 & & \\
\hline
\end{tabular}

\section{B-Diffusion study:}

Diffusion MR imaging was performed before the dynamic study using respiratory triggered fatsuppressed single-shot spin echo echoplanar sequence that combined the two diffusion (motionprobing) gradients before and after the $180^{\circ}$ pulse. The acquisition parameters for 1 Tesla machines were: TR $2270 \mathrm{msec}$, TE $78 \mathrm{msec}$, matrix $100 \times 83$ with a field of view as small as possible, slice thickness $10 \mathrm{~mm}$, slice gap 1-2mm, scan time 4$5 \mathrm{~min}$. We used b values of 0,400 and $800 \mathrm{~s} / \mathrm{mm}^{2}$ The acquisition parameters for 1.5 Tesla machines were: TR $1700 \mathrm{msec}$, TE $76 \mathrm{msec}$, matrix 120 X 95 with a field of view as small as possible, slice thickness $10 \mathrm{~mm}$, slice gap $1-2 \mathrm{~mm}$, scan time 3$4 \mathrm{~min}$. We used b-values of 0,500 and $1000 \mathrm{~s} / \mathrm{mm}^{2}$.

\section{Quantitative diffusion analysis (ADC measure-} ment):

ADC maps were generated on the workstation. Calculation of the ADC value is an automated process available on the workstation. The ROI included the entire embolized area. In case of focal hyperintensity within or at the margins of the embolized lesion; its ADC value was calculated. The ADC was measured three times and the three measurements were averaged.

\section{C- Dynamic study:}

Dynamic study was performed after manual bolus injection of $0.1 \mathrm{mmol} / \mathrm{kg}$ body weight of $\mathrm{Gd}$ DTPA. Dynamic imaging using 3D fat-suppressed T1-weighted gradient echo sequence (THRIVE i.e. T1 high resolution isotropic volume examination). 
A dynamic series consisted of one pre contrast series followed by four successive post contrast series including early arterial, late arterial, and portal phases with 19-21 seconds intervals (17 seconds for image acquisition with breath-holding and 2-4 seconds for re-breathing) this is followed by 5 -min delayed phase imaging. All patients were imaged in end expiration to limit the risk of image misregistration. Acquisition parameters for open 1 Tesla machine were TR $3.3 \mathrm{msec}$., TE $1.6 \mathrm{msec}$., flip angle $10^{\circ}$, matrix size, $136 \times 108$, field of view $300-350 \mathrm{~mm}$ and slice thickness $2-3 \mathrm{~mm}$. Acquisition parameters for 1.5 Tesla machines were TR $4.4 \mathrm{msec}$., TE $2.1 \mathrm{msec}$, flip angle $10^{\circ}$, matrix size, $172 \times 163$, field of view $300-350 \mathrm{~mm}$ and slice thickness $2-3 \mathrm{~mm}$.

\section{Dynamic study analysis:}

Arterial phase enhancement: That should be confirmed by the subtraction images (to prove that the high signal in the arterial phase is due to enhancement and not due to the original pre-contrast high T1 signal). Contrast wash out: Decrease in the enhancement on delayed phase imaging compared with early phase imaging.

\section{MR images were analyzed for the following:}

The morphological features of each lesion were recorded included size, border, signal characteristics at T1, T2 and fat saturated signal characteristics. Pattern of enhancement in the dynamic \& subtracted images. Signal intensity on diffusion images with $\mathrm{ADC}$ values.

\section{Analysis of the MR images:}

Images were sent to the workstation (Phillips Extended MR Workspace) for further image processing. The morphological features of each lesion were recorded including size, border and signal intensity at T1, T2 and SPAIR images. Assessment for the presence of complications, residual or recurrent tumor viability.

\section{Statistical analysis:}

Computer software package SPSS version 15 was used in the analysis. For quantitative variables, mean (as a measure of central tendency), standard deviation (as measures of variability) were presented. Frequency and percentages were presented for qualitative variables. Chi-Square test was used to estimate differences in qualitative variables. A ROC (Receiver Operating Characteristic) curve was constructed and the area below the ROC curve was used to represent prediction precision. $p$-value less or equal to 0.05 was considered significant and less than 0.01 was considered highly significant.

\section{Results}

80 patients were enrolled in this study, 52 were males $(65 \%)$ and 28 were females $(35 \%)$. Age of these patients ranged from 45 to 81 years (mean age: 60 ). We classified the patients into two groups: Resolved (benign) group: No MRI signs of residual or recurrent viability at the treated lesion (regardless the presence of other lesions). Unresolved (malignant) group: If there is evidence of residual or recurrent viability.

46 patients $(57.5 \%)$ have resolved lesions while 34 patients $(42.5 \%)$ have residual or recurrent lesions. The size of chemoembolized lesions ranged from $(1.5$ to $6 \mathrm{~cm})$ the mean and standard deviation for benign group $(2.8 \pm 1.70) \mathrm{cm}$ while malignant $(3.52 \pm 1.30) \mathrm{cm}$. In benign group; $2(4.3 \%)$ was low, $6(13 \%)$ was isointense, $21(45.6 \%)$ was high and $17(39.9 \%)$ was heterogenous signal on T1 WI while $21(45.6 \%)$ was low, $3(19.5 \%)$ was isointense, 5 (10.8\%) was high and 17 (39.9\%) was heterogenous signal on T2 WI series.

In malignant group; 17 (50\%) was low, 7 $(20.5 \%)$ was isointense, $2(2.9 \%)$ was high and 8 $(23.5 \%)$ was heterogenous on T1 WI series while $2(5.8 \%)$ was low, $4(11.7 \%)$ was isointense, 20 $(58.8 \%)$ was high and $8(23.5 \%)$ was heterogenous on T2 WI series.

The border of benign conditions and malignant residuals were classified into smooth, irregular and nodular, 39 patients ( $84.7 \%$ ) of benign group were smooth compared to 17 (50\%) of malignant group while $4(8.6 \%)$ patients of benign group were nodular as compared to $11(32.3 \%)$ patients of malignant group while 6 patients of both benign and malignant groups were irregular $(13 \%) \&$ $(17.6 \%)$ respectively.

\section{Dynamic study interpretation:}

Malignant group: Arterial phase enhancement that should be confirmed by the subtraction images to prove that the high signal in the arterial phase is due to enhancement and not due to the original pre-contrast high $\mathrm{T} 1$ signal) with washout of the contrast in the delayed phase. Benign group: Suggested when no appreciable, progressive or persistent enhancement was detected on dynamic images. Upon correlation of the dynamic findings to final diagnoses, 3 cases were found to be false negative $(3.75 \%), 2$ cases were false positive $(2.5 \%), 45$ were true negative $(56.25 \%)$ and 30 were true positive $(37.5 \%)$ shown in (Table 2 ). 
Based on the previous findings, dynamic MRI had a sensitivity of $90.9 \%$, specificity of $95.7 \%$, positive predictive value of $93.7 \%$, negative predictive value of $93.7 \%$ and overall agreement of $94 \%$. Regarding two false positive results, noted perilesional early arterial enhancement with wash out on delayed phase and becoming isointense to surrounding liver was observed. So, it was misdiagnosed as a residual tumor. Yet, on repeated angiography, no blush was detected. Follow-up CT after 3 months was free. Upon reviewing the MRI of the patient, the arterially enhancing area was isointense on delayed phase of dynamic MRI besides no corresponding signal intensity changes were noted on T1, T2 and STIR weighted images and also showing facilitated diffusion; findings were in favour of chemoembolization induced inflammation and granulation tissue after local treatment.

Regarding three false negative results related to inadequate breath hold by the patient led to motion artifact with subsequent misinterpretation of the perilesional residual lesion as benign reactive change. On follow-up study, the suspected region increased in size denoting residual tumoral viability.

\section{Diffusion MRI study interpretation:}

Viable tumor or malignant group showing restricted diffusion manifested by sustained high signal on diffusion proper and low signal on ADC map images while benign group showing low signal or mild high signal in diffusion proper and high signal on ADC map images (shine through effect). Upon correlation of the diffusion MRI findings to final diagnoses, 16 cases were false positive (20\%), 30 were true negative $(37.5 \%), 34$ were true positive $(42.5 \%)$ and no false negative result seen and that shown in the following (Table 3 ).

Based on the previous findings, diffusion MRI had a sensitivity of $100 \%$, a specificity of $65.2 \%$, a positive predictive value of $68 \%$, and a negative predictive value of $100 \%$ and overall agreement of $80 \%$. The sensitivity, specificity, PPV \& NPV as well as overall agreement for both dynamic and diffusion MRI were calculated and shown in the following (Table 4).

Therefore, addition of DWI to the conventional MR images improves the sensitivity, but decreased the specificity and PPV as it increases the false positive cases.
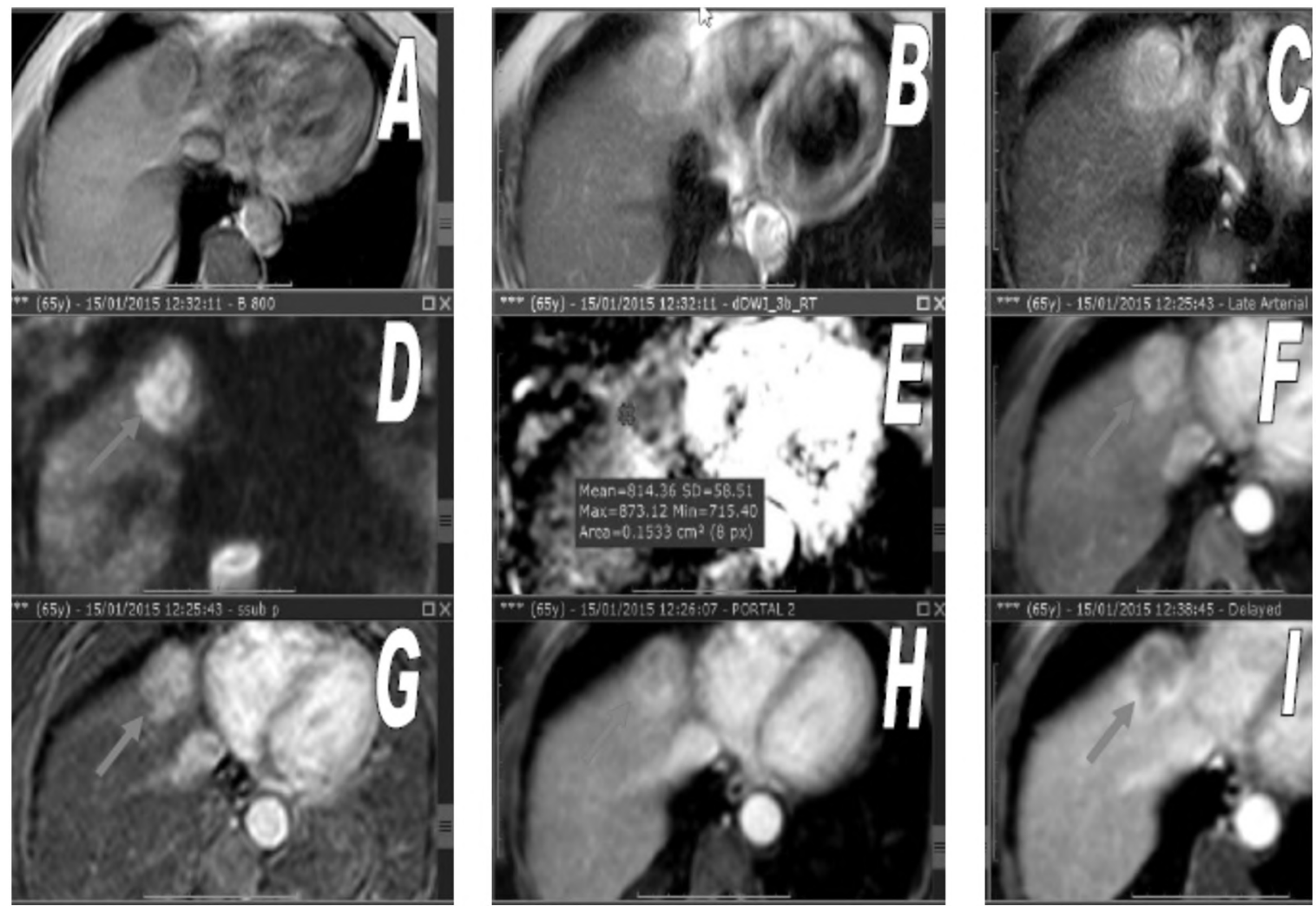

Fig. (1): 65 years old male patient with liver cirrhosis underwent TACE of left hepatic lobe segment IVa focal lesion. The patient underwent dynamic MRI with diffusion study one month after chemoembolization. (A, B \& C) axial T1, T2 \& SPAIR WI one month after the embolization showing treated (segment IVa) lesion displaying low T1 and high T2 and SPAIR signal. (D) Diffusion WIs and (E) ADC map at the same level display diffusion restriction with low ADC value. (F, G, H \& I) dynamic post contrast arterial, arterial subtraction, portal and delayed images showing early arterial enhancement with rapid washout of the contrast on the portal \& delayed phases of the study denoting residual tumoral activity (arrows). 

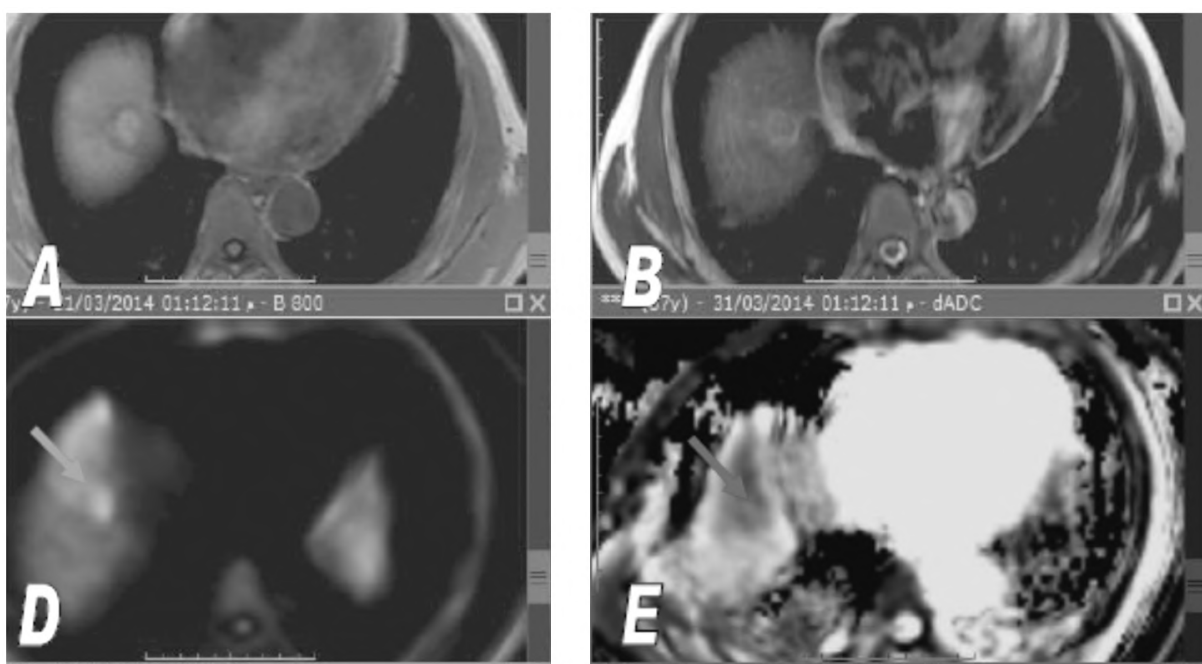

마
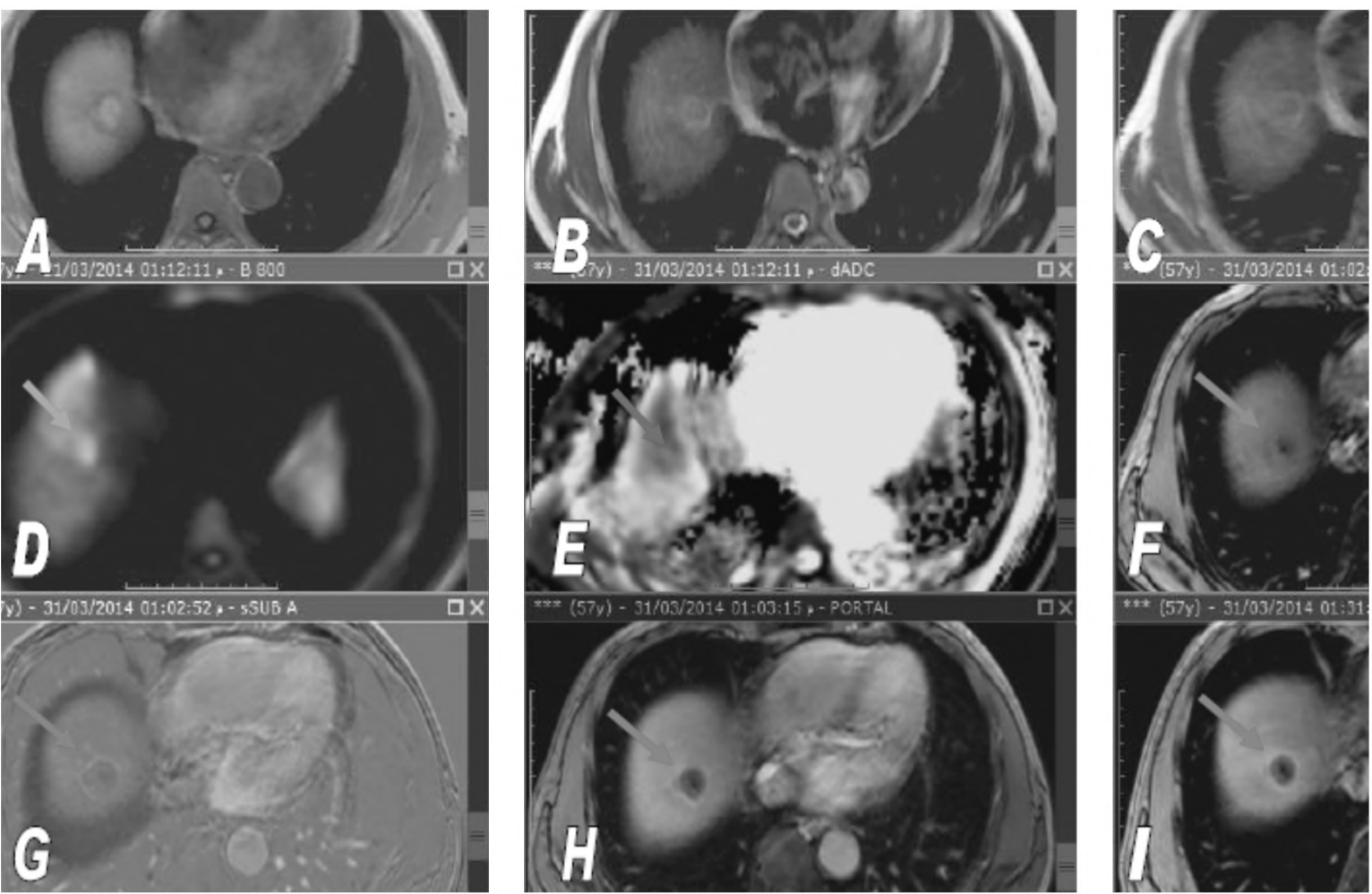

Fig. (2): 57-Year-old female patient with liver cirrhosis underwent TACE of right hepatic lobe segment VIII focal lesion. The patient underwent dynamic MRI with diffusion study $1 \& 3$ months after the chemoembolization. (A, B \& C) axial T1, T2 $\&$ SPAIR WI 1 month after the embolization showing treated segment VIII focal lesion presenting high T1, T2 and SPAIR signal. (D) Diffusion WIs and (E) ADC map at the same level show no significant diffusion restriction. (F, G, H \& I) dynamic post contrast arterial, arterial subtraction, portal and delayed images showing no significant post contrast enhancement on the different phases of the study denoting successful embolization (arrows). Marginal delayed enhancing rim noted denoting granulation tissue.
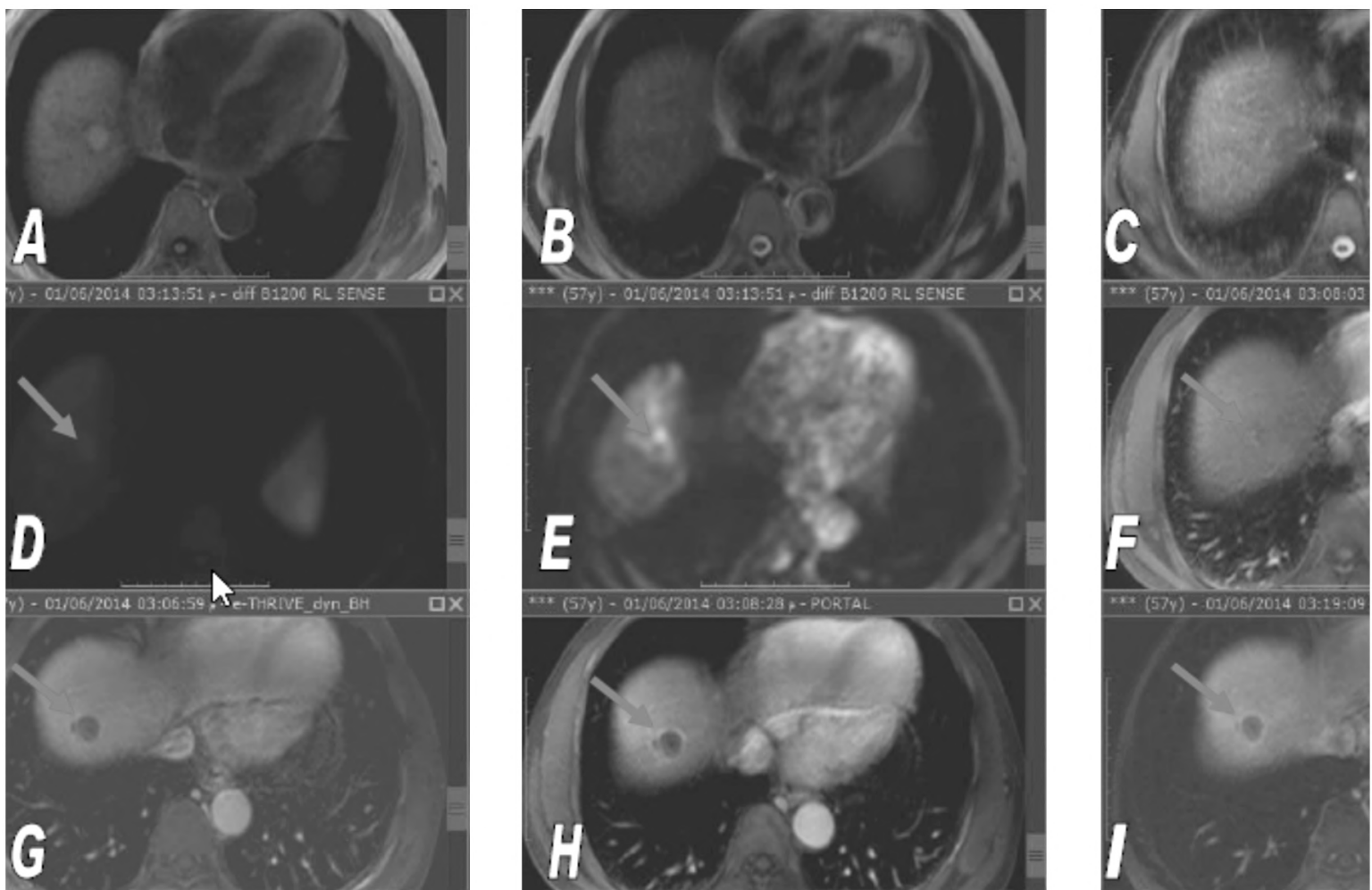

Fig. (3): (Follow-up 3 months after intervention): (A, B \& C) axial T1, T2 \& SPAIR WI showing segment VIII focal lesion currently displaying high T1, intermediate to low T2 and SPAIR signal. (D) Diffusion WIs and (E) ADC map at the same level still show no significant diffusion restriction. (F, G, H \& I) dynamic post contrast arterial, arterial subtraction, portal and delayed images still showing no significant post contrast enhancement on the different phases of the study denoting successful embolization (arrows). Still noted marginal delayed enhancing rim denoting granulation tissue. 
Table (2): Correlating dynamic MRI results to the final diagnosis in the studied group.

\begin{tabular}{lcc}
\hline Dynamic results & Number of lesions & Percentage $\%$ \\
\hline False negative & 3 & $3.75 \%$ \\
False positive & 2 & $2.5 \%$ \\
True negative & 45 & $56.2 \%$ \\
True positive & 30 & $37.5 \%$ \\
\hline Total & 80 & $100 \%$ \\
\hline
\end{tabular}

Table (3): Correlating diffusion MRI results to the final diagnosis in the studied group.

\begin{tabular}{lcc}
\hline Diffusion results & Number of lesions & Percentage $\%$ \\
\hline False negative & 0 & $0 \%$ \\
False positive & 16 & $20 \%$ \\
True negative & 30 & $37.5 \%$ \\
True positive & 34 & $42.5 \%$ \\
\hline Total & 80 & $100 \%$ \\
\hline
\end{tabular}

Table (4): Different indices of dynamic and diffusion MRI.

\begin{tabular}{lcc}
\hline Items & Dynamic MRI & Diffusion MRI \\
\hline Sensitivity & $90.9 \%$ & $100 \%$ \\
Specificity & $95.7 \%$ & $65.2 \%$ \\
PPV & $93.7 \%$ & $68 \%$ \\
NPV & $93.7 \%$ & $100 \%$ \\
Overall agreement & $94 \%$ & $80 \%$ \\
\hline
\end{tabular}

\section{Discussion}

Assessment of tumor response to trans-arterial chemoembolization of hepatocellular carcinoma patients is the corner stone in the management as early favorable response generally indicates effectiveness of therapy, and may result in significant survival benefit. Early identification of treatment failure is also critical in patient management, since a repeat treatment cycle can be performed before disease progression occurs. Among the 80 patients included in our study, 25 patient (32\% of lesions) displayed heterogeneous signal intensity that makes assessment of HCC necrosis after TACE depending only on signal intensity of the pre-contrast sequences a conflict issue. We agreed with Vossen et al., [5] who stated that in addition to residual tumor, T2 hyperintensity can represent liquefactive necrosis, hemorrhage, or inflammatory reaction. In our study five lesions $(10 \%)$ of the benign group demonstrated high T2 signal intensity. Braga et al., [6] stated that dynamic MRI for unsuccessful TACE treatment shows focal enhanced regions after contrast administration admixed within necrotic regions. However, the perilesional hypervascularity detected by dynamic MRI is not specific only for the recurrent lesions but also occur in benign conditions as a result of chemoembolization induced inflammation, granulation tissue or other non tumorous arterioportal shunts [7].

Yu et al., [8] stated that benign conditions would show a perilesional rind-like contour and hyperintensity on delayed phase images and could be distinguished from a more nodular contour and washout of contrast material in residual lesions. In our study, we had relatively lower sensitivity of dynamic MRI compared to other studies done by $\mathrm{Yu}$ et al., [8] and Goshima et al., [9] as we had three cases in which in-appropriate breath holding led to motion artifact with subsequent false negative interpretation by the viewer. We also had two false positive cases due to misinterpretation of a perilesional post interventional reactive changes.

Geschwind et al., [10] reported that viable tumor cells have intact membranes restrict water diffusion in contrast to necrotic tumors which have increased water diffusion due to cell membrane disruption. The ADC reflects the intra and extracellular motion of water molecules in biological tissue and thus supplies information about the tumor microenvironment.

Hayashida et al., [11] and DeVries et al., [12] demonstrated that diffusion-weighted imaging has been used to differentiate between viable and necrotic tumor tissues and to monitor the effect of several treatment options. Buijs et al., [13] and Vossen et al., [5] confirmed that individuals with HCC who responded to chemoembolization treatment reveled a significant increase in the ADC values after therapy.

In our study, the difference between ADC variables between the malignant and benign groups were statistically significant $p$-value 0.006 . The best cut off that maximizes sensitivity and specificity is $1.05 \times 10^{-3} \mathrm{~mm} / \mathrm{s}$. At this ADC value, the sensitivity is 0.95 and specificity is 0.41 . We partially agreed with the studied done by $\mathrm{Yu}$ et al., [8] and Goshima et al., [9], who stated that DWMRI is not a reliable predictor of local HCC recurrence after TACE as compared with gadoliniumenhanced MR imaging.

In the study of Yu et al., [8], DWI increased the sensitivity for determining perilesional tumor recurrence according to the increased confidence level for the sustaining hyperintensity with increasing $b$ factors. However, the number of false positives increased by adding DWI. Although, the decreased specificity compromised the increased 
sensitivity achieved by adding DWI and there is a decrease in the overall diagnostic accuracy.

In our study, we also found that diffusion MRI increased the sensitivity of local HCC detection on the expense of examination specificity due to increased false positives.

Yu et al., [8] stated that hypercellularity intermingled with a fibrotic component in the inflammatory granulation tissue originated from perilesional parenchymal insults could restrict water diffusion, resulting in sustaining hyperintensity on DWI leading to increase in false positive findings.

In our study, 16 false positive cases out of 80 patients were misdiagnosed on diffusion-weighted imaging. When we reviewed the corresponding pattern of diffusion restriction, $76.4 \%$ of true positive cases exhibited focal peripheral nodular restriction, meanwhile $62.5 \%$ of the false positive cases exhibited intra-lesional heterogenous restriction. So, we considered that the increase in false positive findings in our study is likely arising from intra-lesional hemorrhage or liquefactive necrosis. In the studies of Holtas et al., [14] and Tung et al., [15], sterile liquefactive necrosis and intra-cavitary microhaemorrhage are accepted to be the cause of hyperintensity in diffusion-weighted MR images of post therapeutic necrosis of malignant lesions.

Our results are matching with those of $\mathrm{Yu}$ et al., [8] and Goshima et al., [9] showing dynamic contrast enhanced MRI to be superior to diffusion weighted MRI in evaluating HCC response to treatment as dynamic MRI had a sensitivity of $90.9 \%$, a specificity of $95.7 \%$, a positive predictive value of $93.7 \%$, a negative predictive value of $93.7 \%$ and overall agreement of $94 \%$ compared to $100 \%, 65.2 \%, 68 \%, 100 \%$ and $80 \%$ respectively of diffusion weighted imaging.

According to Thoeny et al., [16], diffusion weighted MRI has some advantages compared to dynamic MRI. First, contrast medium administration is not required, and the examination is obtained in a relatively short time. Second, the technique is easy to be repeated, allowing close follow-up during and after tumor treatment. Also, image postprocessing is less time-consuming compared to dynamic contrast enhanced MR imaging. At last, diffusion-weighted MR imaging allows easy evaluation of the whole tumor especially being beneficial in cases of inhomogeneity that may occur within tumors.

In our study, we had some diagnostic limitations. First, it was difficult to obtain pathologic confirmation in patients who underwent chemoembolization because all of these patients were not subjected to surgery. Second, lesions included in our study were larger than $1 \mathrm{~cm}$ in diameter, and few lesions were located at the hepatic dome. The formentioned factors could cause selection bias leading to increased sensitivity of diffusion weighted images since hepatic lesions close to the diaphragm pose a challenge to DW-MRI evaluation as they are more sensitive to motion and susceptibility artifacts.

\section{Conclusion:}

MRI play a pivotal role in assessment tumor response after trans-arterial chemoembolization of hepatocellular carcinoma patients and imaging protocol must include both diffusion and dynamic MRI imaging series DW MR imaging is a rapid promising technique for the non-invasive evaluation of tumor response after TACE particularly when contrast medium administration is contraindicated. Dynamic study is the corner stone in detection of recurrent lesions.

\section{References}

1- KLOECKNER R., OTTO G., BIESTERFELD S., OBERHOLZER K., DUEBER C. and PITTON M.B.: MDCT versus MRI assessment of tumor response after transarterial chemoembolization for the treatment of hepatocellular carcinoma. Cardiovasc. Intervent Radiol., 33 (3): $532-40,2010$

2- KIM S., MANNELLI L., HAJDU C.H., BABB J.S., CLARK T.W., HECHT E.M., et al.: Hepatocellular carcinoma: Assessment of response to transarterial chemoembolization with image subtraction. J. Magn. Reson. Imaging, 31 (2): 348-55, 2010

3- EISENHAUER E.A., THERASSE P., BOGAERTS J., SCHWARTZ L.H., SARGENT D., FORD R., et al.: New response evaluation criteria in solid tumours: Revised RECIST guideline (version 1.1). Eur. J. Cancer, 45 (2): 228-47, 2009.

4- OZKAVUKCU E., HALILOGLU N. and ERDEN A.: Post-treatment MRI findings of hepatocellular carcinoma. Diagn. Interv. Radiol., 15 (2): 111-20, 2009.

5- VOSSEN J.A., BUIJS M. and KAMEL I.R.: Assessment of tumor response on MR imaging after locoregional therapy. Tech. Vasc. Interv. Radiol., 9 (3): 125-32, 2006.

6- BRAGA L., GULLER U. and SEMELKA R.C.: Pre-, peri-, and posttreatment imaging of liver lesions. Radiol. Clin. North Am., 43 (5): 915-27, viii, 2005.

7- YU J.S.: Hepatocellular carcinoma after transcatheter arterial chemoembolization: Difficulties on imaging follow-up. Korean J. Radiol., 6 (3): 134-5, 2005.

8- YU J.S., KIM J.H., CHUNG J.J. and KIM K.W.: Added value of diffusion-weighted imaging in the MRI assessment of perilesional tumor recurrence after chemoembolization of hepatocellular carcinomas. J. Magn. Reson. Imaging, 30 (1): 153-60, 2009. 
9- GOSHIMA S., KANEMATSU M., KONDO H., YOKOYAMA R., KAJITA K., TSUGE Y., et al.: Diffusion-weighted imaging of the liver: Optimizing $b$ value for the detection and characterization of benign and malignant hepatic lesions. J. Magn. Reson. Imaging, 28 (3): 691-7, 2008.

10- GESCHWIND J.F., ARTEMOV D., ABRAHAM S., OMDAL D., HUNCHAREK M.S., McGEE C., et al.: Chemoembolization of liver tumor in a rabbit model: Assessment of tumor cell death with diffusion-weighted MR imaging and histologic analysis. J. Vasc. Interv. Radiol., 11 (10): 1245-55, 2000.

11-HAYASHIDA Y., YAKUSHIJI T., AWAI K., KATAHIRA K., NAKAYAMA Y., SHIMOMURA O., et al.: Monitoring therapeutic responses of primary bone tumors by diffusion-weighted image: Initial results. Eur. Radiol., 16 (12): 2637-43, 2006.

12- DeVRIES A.F., KREMSER C., HEIN P.A., GRIEBEL J., KREZCY A., OFNER D., et al.: Tumor microcirculation and diffusion predict therapy outcome for primary rectal carcinoma. Int. J. Radiat. Oncol. Biol. Phys., 56 (4): 958 65, 2003.
13- BUIJS M., VOSSEN J.A., HONG K., GEORGIADES C.S., GESCHWIND J.F. and KAMEL I.R.: Chemoembolization of hepatic metastases from ocular melanoma: Assessment of response with contrast-enhanced and diffusion-weighted MRI. AJR Am. J. Roentgenol., 191 (1): 285-9, 2008.

14- HOLTAS S., GEIJER B., STROMBLAD L.G., MALYSUNDGREN P. and BURTSCHER I.M.: A ring-enhancing metastasis with central high signal on diffusion-weighted imaging and low apparent diffusion coefficients. Neuroradiology, 42 (11): 824-7, 2000.

15- TUNG G.A., EVANGELISTA P., ROGG J.M. and DUNCAN J.A., 3 rd: Diffusion-weighted MR imaging of rimenhancing brain masses: Is markedly decreased water diffusion specific for brain abscess? AJR Am. J. Roentgenol., 177 (3): 709-12, 2001.

16- THOENY H.C., De KEYZER F., CHEN F., NI Y., LANDUYT W., VERBEKEN E.K., et al.: Diffusion-weighted MR imaging in monitoring the effect of a vascular targeting agent on rhabdomyosarcoma in rats. Radiology, 234 (3): 756-64, 2005.

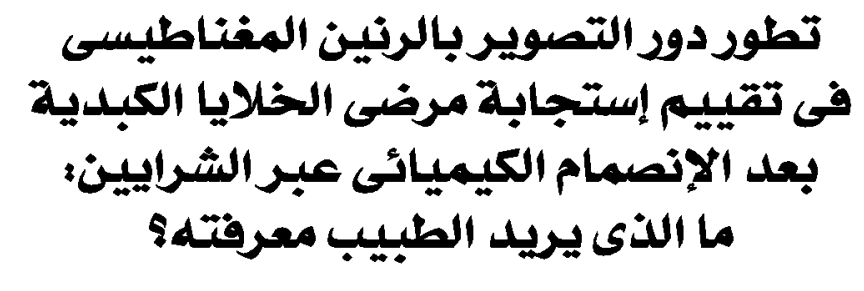

سرطان الخلايا الكبدية هو خامس آكثر آنواع الآقدام الخبيثة شيوعاً في العالم والثالث الآكثر شيوعاً للوفاة بالسرطان . يستخدم الصقن

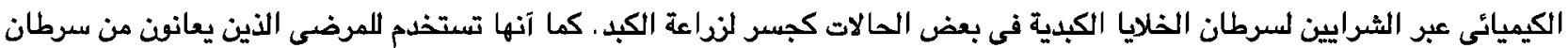

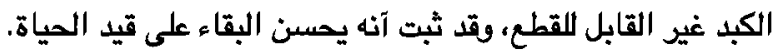

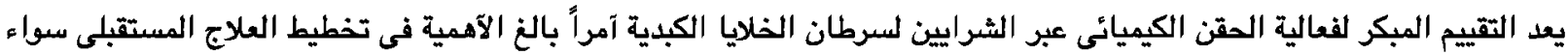

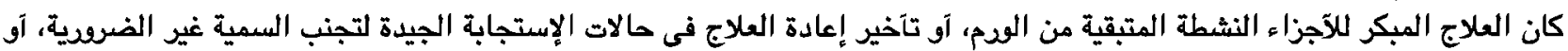

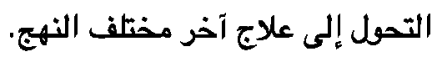

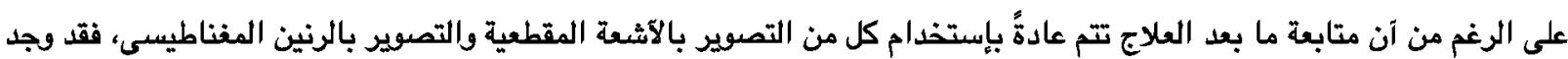

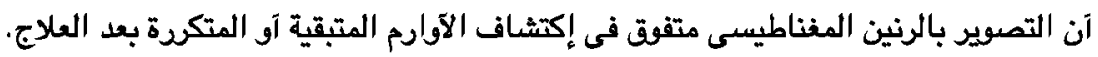

تشمل مزايا التصوير بالرنين المغناطيسى فى التحقيق فى آمراض الكبد عدم وجود إشعاع مؤين وإن مخلبات الجابولينينيوم آقل عرضية

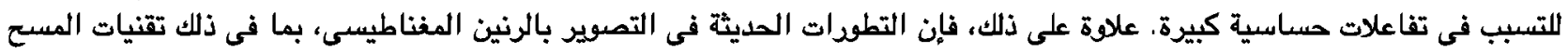

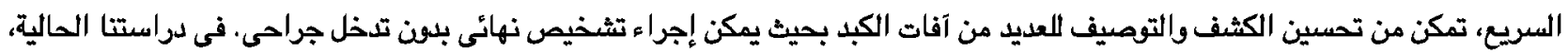

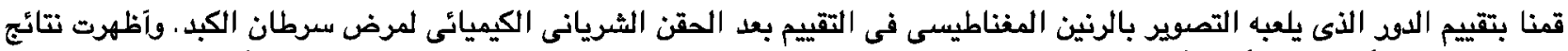

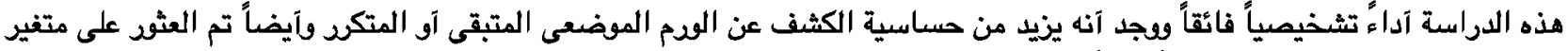

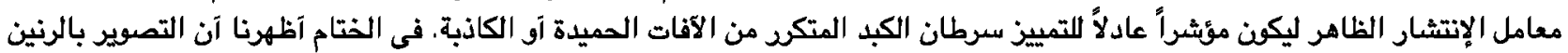

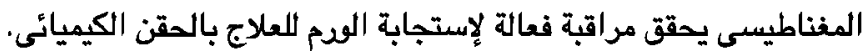

